\title{
Therapeutic temperature modulation in severe or moderate traumatic brain injury: a propensity score analysis of data from the Nationwide Japan Neurotrauma Data Bank
}

\author{
Kei Miyata, MD, ${ }^{1,2,5}$ Hirofumi Ohnishi, MD, PhD, ${ }^{3}$ Kunihiko Maekawa, MD, ${ }^{4}$ \\ Takeshi Mikami, MD, PhD, ${ }^{2}$ Yukinori Akiyama, MD, PhD, ${ }^{2}$ Satoshi lihoshi, MD, PhD, ${ }^{2}$ \\ Masahiko Wanibuchi, MD, PhD, ${ }^{2}$ Nobuhiro Mikuni, MD, PhD, ${ }^{2}$ Shuji Uemura, MD, PhD, ${ }^{1}$ \\ Katsutoshi Tanno, MD, PhD, ${ }^{1}$ Eichi Narimatsu, MD, PhD, ${ }^{1,5}$ and Yasufumi Asai, MD, PhD ${ }^{1,5}$ \\ Departments of ${ }^{1}$ Emergency Medicine, ${ }^{2}$ Neurosurgery, and ${ }^{3}$ Public Health, Sapporo Medical University; ${ }^{4}$ Emergency and \\ Critical Care Center, Hokkaido University Hospital, Sapporo; and ${ }^{5}$ Japan Neurotrauma Data Bank Committee, Japan Society of \\ Neurotraumatology, Tokyo, Japan
}

OBJECTIVE In patients with severe traumatic brain injury (TBI), a randomized controlled trial revealed that outcomes did not significantly improve after therapeutic hypothermia $(\mathrm{TH})$ or normothermia $(\mathrm{TN})$. However, avoiding pyrexia, which is often associated with intracranial disorders, might improve clinical outcomes. The objective of this study was to compare neurological outcomes among patients with moderate and severe TBI after therapeutic temperature modulation (TTM) in the absence of other interventions.

METHODS Data from 1091 patients were obtained from the Japan Neurotrauma Data Bank Project 2009, a cohort observational study. Patients with cardiac arrest, those with a Glasgow Coma Scale score of 3 and dilated fixed pupils, and those whose cause of death was injury to another area of the body were excluded, leaving 687 patients aged 16 years or older in this study. The patients were divided into 2 groups: the TTM group underwent TN (213 patients) or TH (82 patients), and the control group (392 patients) did not receive TTM. The primary end point for this study was the rate of poor outcome at hospital discharge, and the secondary end point was in-hospital death. Out of the 208 total items in the database, 29 variables that could potentially affect outcome were matched using the propensity score (PS) method in order to reduce selection bias and balance the baseline characteristics.

RESULTS From each group, 141 patients were extracted using the PS-matching process. Among the patients in the TTM group, 29 had undergone TH and 112 had undergone TN. In a log-rank test using Kaplan-Meier survival curves, no significant differences in patient outcome or death were observed between the 2 groups (poor outcome, $p=0.83$; death, $p=0.18$ ). A Cox proportional-hazards regression analysis established the HR for poor outcome and mortality at 1.03 $(95 \% \mathrm{Cl} 0.78-1.36, \mathrm{p}=0.83)$ and $1.34(95 \% \mathrm{Cl} 0.87-2.07, \mathrm{p}=0.18)$, respectively.

CONCLUSIONS There was no clear improvement in neurological outcomes after TTM in patients with moderate or severe TBI. To elucidate the role of TTM in patients with these injuries, a prospective study is needed with long-term follow-up using specific target temperatures.

http://thejns.org/doi/abs/10.3171/2015.3.JNS141895

KEY WORDS therapeutic temperature modulation; traumatic brain injury; propensity; neurocritical care; hypothermia; normothermia

\footnotetext{
ABBREVIATIONS AED = antiepilepsy drug; AIS = Abbreviated Injury Scale; DI = diffuse injury; DNAR = do not attempt resuscitation; eML= evacuated mass lesion; GCS = Glasgow Coma Scale; GOS = Glasgow Outcome Scale; ICP = intracranial pressure; ICU = intensive care unit; ISS = Injury Severity Score; IVH = intraventricular hemorrhage; JNTDB = Japan Neurotrauma Data Bank; NABIS = National Acute Brain Injury Study; PS = propensity score; T\&D = talk and deteriorate; TBI = traumatic brain injury; NMBD = neuromuscular blockage drug; NTCDB = National Traumatic Coma Data Bank; TH = therapeutic hypothermia; $T \mathrm{~N}=$ therapeutic normothermia; $\mathrm{tSAH}=$ traumatic subarachnoid hemorrhage; TTM = therapeutic temperature modulation.

SUBMITTED August 31, 2014. ACCEPTED March 30, 2015.
}

INCLUDE WHEN CITING Published online September 18, 2015; DOI: 10.3171/2015.3.JNS141895. 
$\mathrm{T}$ HE importance of therapeutic temperature modulation (TTM) has been suggested in various areas of intensive care. There is some clinical evidence of benefit from TTM at temperatures between $32^{\circ} \mathrm{C}$ and $34^{\circ} \mathrm{C}$ for comatose adults after cardiopulmonary resuscitation for an initial ventricular fibrillation ${ }^{1}$ or at temperatures between $32.5^{\circ} \mathrm{C}$ and $35.5^{\circ} \mathrm{C}$ for patients with neonatal hypoxic-ischemic encephalopathy., ${ }^{7,12}$ Although TTM is strongly recommended for such patients, no conclusion has been reached regarding the effects of TTM and the most appropriate temperatures..$^{18}$

Nevertheless, in recent years, TTM has been performed even in patients with severe traumatic brain injury (TBI). Experimental studies and small clinical studies of patients with TBI have reported that therapeutic hypothermia $(\mathrm{TH})$ improves oxygen metabolism in brain tissue, ${ }^{27}$ inhibits intracranial hypertension, ${ }^{10}$ and improves patient outcomes; 9,28 these studies recommend the induction of TTM soon after the trauma occurs. However, there is no strong evidence that $\mathrm{TH}$ improves the outcomes of patients with severe TBI. 3,15,22,24,25 Yet given the relationship between pyrexia and intracranial disorders, the assumption that avoiding high body temperature through the use of therapeutic normothermia (TN) can improve prognosis might be valid. ${ }^{13,23}$

The objective of this study was to estimate neurological outcomes in patients with TBI following TTM and conventional management and to compare differences between well-balanced groups, based on a propensity score (PS)-matching method.

\section{Methods \\ Data Collection}

The Japan Neurotrauma Data Bank (JNTDB) Project 2009, a prospective nationwide observational study, was used as the database for this study. Among the 22 medical facilities that contributed data to this project, there were 6 advanced critical care and emergency centers, 13 emergency centers, and 3 emergency neurosurgical hospitals.

The data analyzed in this study came from patients of all ages enrolled in the JNTDB Project 2009, with Glasgow Coma Scale (GCS) scores of 8 or less upon hospital admission or deterioration to that level within 72 hours of trauma. Patients enrolled in the data bank were identified according to the following requirements: 1) all age groups, including children younger than 1 year of age; 2) a GCS score of $\leq 8$ at the time of hospitalization or a condition deteriorating to a GCS score $<8$ within 72 hours of admission; and 3) neurosurgical intervention as treatment for the trauma (excluding chronic subdural hematoma but including bur hole surgery). The enrollment period extended from July 1, 2009, to June 30, 2011. The survey period for prognosis generally lasted 6 months. The datasheet list contained 208 items such as patient characteristics, mechanism of injury, method of transport to the hospital, vital signs at the time of admission, imaging findings, acute-phase treatment, surgical intervention, intracranial pressure (ICP) sensor placement, pharmacotherapy, and methods of TTM. The list also contained Glasgow Outcome Scale (GOS) score (patient outcome at the time of hospital discharge, at the last medical examination, and 6 months after trauma), social rehabilitation condition, and cause of death.

A total of 1091 patients from 22 medical facilities were enrolled; $8 \%$ of the patients were missing data. The number of patients enrolled from each facility varied from 7 to 151. A breakdown of facility types shows that 385 patients were treated at 1 of the 6 advanced critical care and emergency centers, 671 patients at 1 of the 13 emergency centers, and 35 patients at 1 of the 3 neurosurgical hospitals.

\section{Study Design and Patients}

The following patients were excluded from the study: 1) patients with cardiac arrest at the scene of injury or during transport to the hospital; 2) patients for whom the method of TTM was not specified; 3) children with an age $<16$ years; 4) patients with mild TBI, defined as a GCS score of $14-15 ; 5)$ patients with a GCS score of 3 and bilateral fixed and dilated pupils; 6) patients who died from trauma to other parts of the body; and 7) patients who received surgical treatment for a depressed skull fracture, removal of foreign substances from the skull, or penetrating trauma. After these exclusions, 687 patients were available for analysis in this study (Fig. 1).

The patients were divided into a TTM group (295 patients) and a control (C) group (392 patients) based on the treatment they received.

\section{Definition of Temperature Modulation}

In this study, TTM was defined as a temperature management method that uses a noninvasive surface-cooling device (e.g., cooling blankets) to intentionally maintain a certain body temperature, and this definition included both TH and TN. Of the 295 patients in the TTM group, 213 patients underwent TN and 82 underwent TH. For patients undergoing $\mathrm{TN}$, body temperature was maintained below $37^{\circ} \mathrm{C}$. For patients undergoing $\mathrm{TH}$, body temperature was strictly maintained at temperatures below $35^{\circ} \mathrm{C}$. The 392 patients in the $\mathrm{C}$ group did not undergo TTM with any mechanical cooling device but did receive routine medical management including ice-pack cooling or antipyretic drugs, the latter only for patients with a high fever. There is no fixed consensus regarding indications for introducing TTM at a health care facility. The 3 types of temperature management were used as deemed appropriate based on the scale of each institution and patient status.

\section{Statistical Analysis}

As patients were not randomly assigned to the TTM or $\mathrm{C}$ group, the role of selection of neurocritical care as a confounding variable cannot be ignored. Furthermore, it is possible that the standards of treatment differed depending on the capabilities of the various facilities. Thus, large differences in baseline patient characteristics and clinical variables, both potentially affecting outcome, may exist between the groups. To account for this, we developed a PS for TTM and controlled for potential confounding and selection bias. Logistic regression was used to determine the propensity to undergo TTM. Patients were classified 


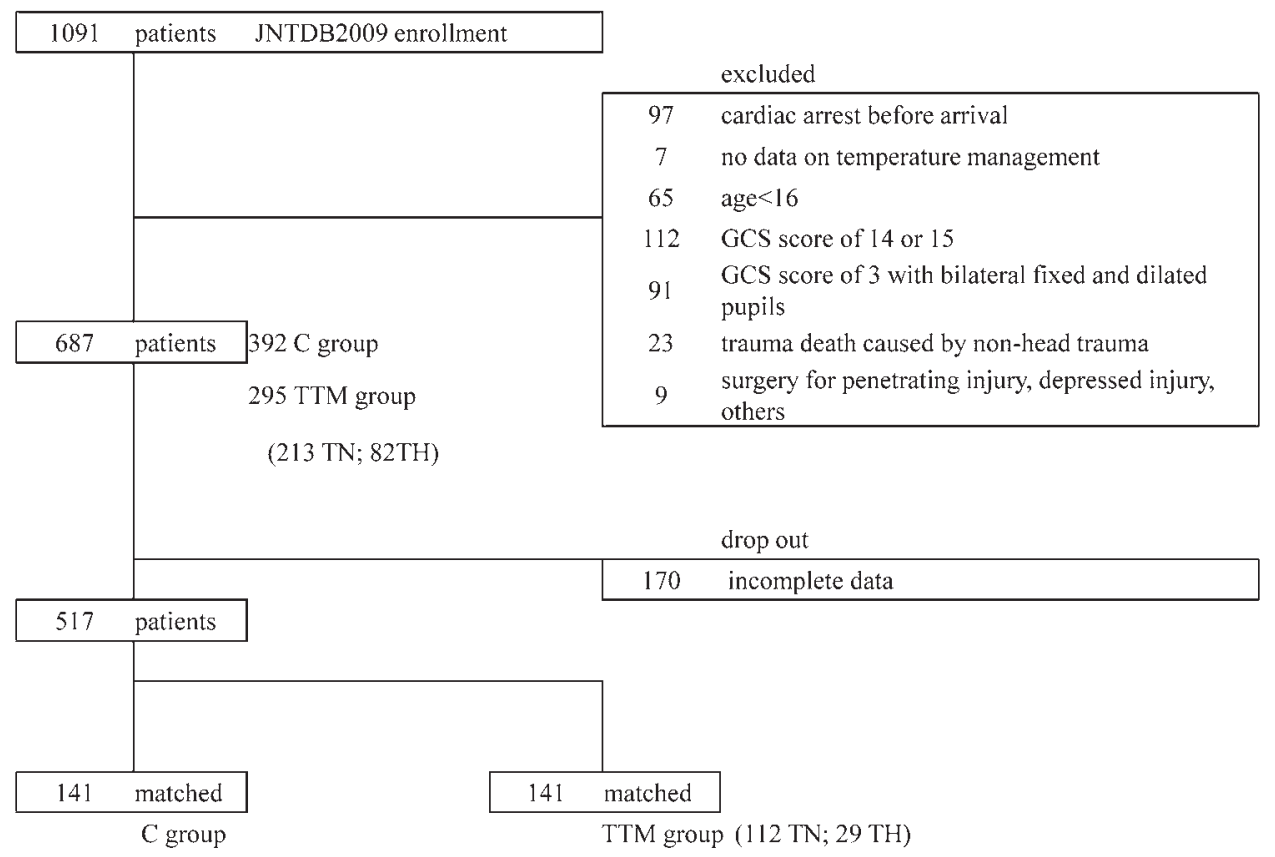

FIG. 1. Study population flow diagram.

in the TTM group if they underwent TN or TH within the acute phase of injury and in the $\mathrm{C}$ group if they did not.

Variables affecting clinical outcomes were extracted: 4 continuous variables and 25 categorical variables associated with patient background such as prehospitalization data, vital signs at hospitalization, neurological findings, radiological findings, neurocritical care, and surgical intervention. Each medical facility independently decided when to begin TTM (e.g., directly after arrival, before or after surgical intervention, or when accelerated intracranial hypertension occurred during intensive care unit [ICU] treatment). Therefore, items concerning neurocritical care treatment and surgical intervention were included as covariates.

Continuous variables included patient age, GCS score upon hospital arrival, head Abbreviated Injury Scale (AIS) score, and Injury Severity Score (ISS). Categorical variables included patient sex, involvement in a traffic accident (yes/no), type of transportation (direct or indirect) to the hospital, time (in minutes) from injury site to hospital arrival (unknown, $<30,31-59$, or $\geq 60$ ), type of medical facility (advanced critical care and emergency center, emergency medical service center, or neurosurgical hospital), vital signs upon arrival, and neurological state upon admission. Neurological state was assessed via respiratory rate per minute $(0-9,10-24$, or $\geq 25)$, systolic arterial blood pressure $(<90$ or $\geq 90 \mathrm{~mm} \mathrm{Hg})$, body temperature upon admission $\left(<35^{\circ}\right.$ or $\left.\geq 35^{\circ} \mathrm{C}\right)$, hypoxia: arterial $\mathrm{pO}_{2}(<80$ or $\geq 80 \mathrm{~mm} \mathrm{Hg})$, lactic acidosis $(\mathrm{pH}<$ 7.2 or $\geq 7.2)$, blood glucose $(<70,70-199,200-299$, or $\geq 300 \mathrm{mg} / \mathrm{dl}$ ), pupil abnormality (none, unilateral, or bilateral), worsening consciousness (i.e., the phenomenon known as "talk and deteriorate" [T\&D], in which patients utter recognizable words at some point after head injury but later deteriorate to a severe head-injured condition
[GCS score $\leq 8$ ] within 72 hours of injury), and radiological findings in the first head CT scan. These radiological findings included the presence of an intraventricular hemorrhage (IVH), traumatic subarachnoid hemorrhage (tSAH), midline shift $(0-4,5-10$, or $>10 \mathrm{~mm})$, basal cisterns (cistern present, compressed, or absent), and final CT classification of diffuse brain injury (DI) according to the National Traumatic Coma Data Bank (NTCDB; Diffuse Injury I or II [DI-I or DI-II], DI-III or DI-IV, evacuated mass lesion [eML], or non-eML). Neurointensive care and surgical interventions were also accounted for, including type of surgery (nonsurgical, bur hole surgery, craniotomy or craniectomy, or others), use of sedative drugs, analgesic drugs, neuromuscular blockage drugs (NMBDs), antiepilepsy drugs (AEDs), diuretic drugs, and ICP sensor placement. Pathology findings in this study were classified into the following 4 groups: mild diffuse injury, severe diffuse injury, eML, and non-eML. Mild diffuse injury included DI-I (no visible intracranial pathology on CT scans) and DI-II (cisterns present and a midline shift of $0-5 \mathrm{~mm}$ and/ or no high- or mixed-density lesions larger than $25 \mathrm{~cm}^{3}$ ). Severe diffuse injury included DI-III (cisterns compressed or absent and a midline shift of $0-5 \mathrm{~mm}$ and/or no highor mixed-density lesions larger than $25 \mathrm{~cm}^{3}$ ) and DI-IV (a midline shift greater than $5 \mathrm{~mm}$ and no high- or mixeddensity lesions larger than $25 \mathrm{~cm}^{3}$ ). Space-occupying lesions larger than $25 \mathrm{~cm}^{3}$ that were surgically excised were considered eML, while non-eML was defined as the presence of space-occupying lesions larger than $25 \mathrm{~cm}^{3}$ that were not surgically removed. All variables were retained in formulating the PS regardless of statistical significance because they were believed to be clinically relevant. Only patients with complete data sets were used in the creation of the PS.

The estimated probability of each patient undergoing 
TTM (the PSs) were then used to match control patients with patients in the TTM group using 1-to-1 matching without replacement. To verify that the matching procedure produced a balanced sample, differences in the propensity model variables (univariate analysis) were tested according to whether TTM was performed on the unmatched sample as well as on the matched-pairs sample. If a good balance was achieved by the matching procedure, greedy matching (nearest-neighbor matching within a caliper [ $\leq 0.03]$ ) was used for PS matching. To calculate the PS, we fit a full nonparsimonious logistic regression model. This model yielded a $\mathrm{C}$ statistic of 0.819 , which indicated a very strong ability to differentiate between patients who were treated with temperature management and those who were not. The PS ranged from 0.04 to 0.97 , which indicated that the probability of undergoing TTM was between 0 and 1. After matching, the groups were examined for any significant differences.

The statistical software package SPSS version 22 (IBM Corp.) was used to analyze the data. An unpaired t-test and a chi-square test were used to compare continuous and categorical variables, respectively, between the 2 groups. After PS matching, the paired t-test and the McNemar test were used.

\section{Primary Outcome and Secondary Outcome}

Clinical outcome was assessed using the GOS. A favorable outcome was defined as a GOS of 5 (good recovery) or 4 (moderate disability), while a poor outcome was defined as a GOS of 3 (severe disability), 2 (persistent vegetative state), or 1 (dead). The primary outcome was defined as the rate of poor outcomes at hospital discharge, and the secondary outcome was the in-hospital death rate. Before and after PS matching, the following statistical methods were used to examine the data. A Kaplan-Meier curve was fitted to estimate both the cumulative favorable outcome rate and the cumulative survival rate at discharge, and a 2 -sided log-rank test was used to perform group comparisons. To estimate the effects of TTM on clinical outcome, a Cox proportional-hazards model was used to calculate a hazard ratio for poor outcome at hospital discharge and for patient mortality, along with $95 \%$ confidence intervals in patients who underwent TTM. After PS matching, a subgroup analysis was performed for patient age (younger or older than 65 years), GCS category at the time of hospitalization (GCS scores of 3-5 = extremely severe, $6-8=$ severe, and 9-13 = moderate), and final CT classification categories, which were related to clinical outcomes. Statistical significance was set at $\mathrm{p}<0.05$.

\section{Results}

\section{Baseline Characteristics and Treatment, and Surgical Intervention}

Before PS matching, large differences were seen between the groups in the categorical and continuous variables (Table 1). Some items had significantly higher values in the TTM group than in the $\mathrm{C}$ group, including a younger patient age, being involved in a traffic accident, direct transport to the hospital, short travel time to the hospital, advanced critical care and emergency center, unilateral mydriasis, hypothermia $\left(<35^{\circ} \mathrm{C}\right)$ at hospital arrival, neurointensive medication (sedative drugs, analgesic drugs, NMBDs, AEDs, diuretic drugs), craniotomy, severe AIS head score, and severe diffuse injury. The only item that had a lower value in the TTM group was bilateral dilation of pupils.

A total of 170 patients were excluded from the study because of missing data; the data from the remaining 517 patients were used for analysis. Data from 141 patients in each group were extracted by PS matching. Of the 141 patients whose data were extracted from the TTM group, 29 patients had undergone TH and 112 had undergone TN (Fig. 1). When patient characteristics were compared between the 2 groups after PS matching, no significant differences related to patient characteristics or treatment interventions were seen (Table 1).

\section{Primary and Secondary Outcomes}

Before PS matching, 491 of 687 patients (71\%) had a poor neurological outcome at discharge, and 231 (34\%) died. The mean follow-up period for all patients was $36 \pm$ 44 days (range 1-311 days). The total number of persondays was 12,834 and 12,048 for the C group and the TTM group, respectively. In the C group, 290 (74\%) patients had a poor neurological outcome and $146(37 \%)$ deaths occurred; in the TTM group, there were 201 (68\%) patients with poor outcome and 85 deaths (29\%). A KaplanMeier curve analysis revealed that neurological outcome ( $\mathrm{p}=0.002$; Fig. 2 left) and survival rate $(\mathrm{p}=0.003$; Fig. 3 left) were significantly better in the TTM group before PS matching. The results of the Cox proportional-hazards analysis (Table 2) indicated that TTM significantly decreased the risk of poor neurological outcome (HR 0.76, 95\% CI $0.63-0.91, p=0.003)$. Furthermore, TTM appeared to significantly lower the risk of death (HR 0.68 , 95\% CI $0.52-0.88, \mathrm{p}=0.004$ ).

In contrast, after PS matching, 171 of 282 patients (61\%) had a poor neurological outcome at hospital discharge, and 83 (29\%) patients died. In the C group, 102 (72\%) patients had a poor outcome and $36(26 \%)$ deaths occurred; in the TTM group, there were 97 (69\%) patients with a poor outcome and 47 deaths (33\%). No significant differences were seen between the 2 groups in neurological outcome at discharge ( $p=0.83$; Fig. 2 right). The cumulative survival rate tended to be high in the $\mathrm{C}$ group $(\mathrm{p}=0.18$; Fig. 3 right). This apparently significant decrease in the risk of primary outcome was not statistically confirmed (HR $1.03,95 \%$ CI $0.78-1.36, \mathrm{p}=0.83$; Table 2). Subgroup analysis (Table 3) revealed that TTM tended to reduce the risk of poor clinical outcomes in patients who underwent the surgical removal of lesions (HR 0.83, 95\% CI 0.57-1.21, $\mathrm{p}=0.33$ ) and in moderate TBI patients (HR $0.72,95 \% \mathrm{CI}$ $0.39-1.32, p=0.29$ ), although these tendencies were not obviously significant. After PS-matching, the HR for mortality changed from 0.68 to 1.34 . An inverse association was observed between TTM and mortality as compared with the result from before PS-matching (Table 2).

\section{Discussion}

In 2001 and 2011, Clifton et al. reported that there were 
TABLE 1. Comparison of categorical and continuous variables for patients in the $\mathrm{C}$ group and TTM group before and after PS matching*

\begin{tabular}{|c|c|c|c|c|c|c|c|}
\hline \multirow[b]{2}{*}{ Parameter } & \multirow[b]{2}{*}{ Total } & \multicolumn{3}{|c|}{ Before PS Matching } & \multicolumn{3}{|c|}{ After PS Matching } \\
\hline & & C Group & TTM Group & $p$ Value & C Group & TTM Group & $\mathrm{p}$ Value \\
\hline No. of patients & & 392 & 295 & & 141 & 141 & \\
\hline Male sex (\%) & $482(70 \%)$ & $272(69 \%)$ & $210(71 \%)$ & 0.61 & $105(75 \%)$ & $98(70 \%)$ & 0.44 \\
\hline Mean age in yrs & - & $65 \pm 19$ & $54 \pm 21$ & $<0.001$ & $60 \pm 21$ & $60 \pm 21$ & 0.69 \\
\hline Age $\geq 65$ yrs & $349(51 \%)$ & $236(60 \%)$ & $113(38 \%)$ & $<0.001$ & $70(50 \%)$ & $74(53 \%)$ & 0.94 \\
\hline Traffic accident & $270(39 \%)$ & $136(35 \%)$ & $134(46 \%)$ & 0.004 & $54(38 \%)$ & $51(36 \%)$ & 0.90 \\
\hline Direct transportation & $508(74 \%)$ & $274(70 \%)$ & $234(79 \%)$ & 0.005 & $104(74 \%)$ & $109(77 \%)$ & 0.53 \\
\hline \multicolumn{8}{|l|}{ Time from injury to hospital } \\
\hline Unknown & $89(13 \%)$ & $71(18 \%)$ & $18(6 \%)$ & $<0.001$ & $18(13 \%)$ & $8(6 \%)$ & 0.06 \\
\hline$<30 \mathrm{~min}$ & $73(11 \%)$ & $28(7 \%)$ & $45(15 \%)$ & 0.001 & $11(8 \%)$ & $20(14 \%)$ & 0.14 \\
\hline $31-59 \mathrm{~min}$ & $237(35 \%)$ & $117(30 \%)$ & $120(41 \%)$ & 0.003 & $51(36 \%)$ & $54(38 \%)$ & 0.80 \\
\hline$\geq 60 \mathrm{~min}$ & $288(42 \%)$ & $176(45 \%)$ & $112(38 \%)$ & 0.007 & $61(43 \%)$ & $59(42 \%)$ & 0.90 \\
\hline \multicolumn{8}{|l|}{ Type of facility } \\
\hline Advanced emergency center & $315(46 \%)$ & $144(37 \%)$ & $171(58 \%)$ & $<0.001$ & $72(51 \%)$ & $65(46 \%)$ & 0.47 \\
\hline Emergency center & $348(51 \%)$ & $231(59 \%)$ & $117(40 \%)$ & $<0.001$ & $64(45 \%)$ & $73(52 \%)$ & 0.34 \\
\hline Neurosurgical hospital & $24(4 \%)$ & $17(4 \%)$ & $7(2 \%)$ & 0.17 & $5(4 \%)$ & $3(2 \%)$ & 0.73 \\
\hline \multicolumn{8}{|l|}{ RR per min } \\
\hline $0-9$ & $6(1 \%)$ & $3(1 \%)$ & $3(1 \%)$ & 1 & $1(1 \%)$ & $2(2 \%)$ & 1 \\
\hline $10-24$ & $489(75 \%)$ & $278(76 \%)$ & $211(73 \%)$ & 0.42 & $101(76 \%)$ & $105(77 \%)$ & 1 \\
\hline$\geq 25$ & $161(25 \%)$ & $86(23 \%)$ & $75(26 \%)$ & 0.46 & $31(23 \%)$ & $20(22 \%)$ & 0.88 \\
\hline Systolic ABP <90 mm Hg & $26(4 \%)$ & $17(4 \%)$ & $9(3 \%)$ & 0.38 & $6(4 \%)$ & $5(4 \%)$ & 1 \\
\hline Body temperature $<35^{\circ} \mathrm{C}$ & $78(12 \%)$ & $34(9 \%)$ & $44(15 \%)$ & 0.02 & $11(8 \%)$ & $16(12 \%)$ & 0.42 \\
\hline Hypoxia, $\mathrm{pO}_{2}<80 \mathrm{~mm} \mathrm{Hg}$ & $71(12 \%)$ & $41(13 \%)$ & $30(11 \%)$ & 0.56 & $15(12 \%)$ & $19(15 \%)$ & 0.23 \\
\hline Lactic acidosis, pH <7.2 & $19(3 \%)$ & $8(2 \%)$ & $11(4 \%)$ & 0.26 & $3(2 \%)$ & $3(2 \%)$ & 1 \\
\hline \multicolumn{8}{|l|}{ Blood glucose level (mg/dl) } \\
\hline$<70$ & $2(0.3 \%)$ & $2(0.5 \%)$ & $0(0 \%)$ & 0.51 & $1(1 \%)$ & $0(0 \%)$ & 1 \\
\hline 70-199 & $481(72 \%)$ & $270(71 \%)$ & $211(73 \%)$ & 0.72 & $102(73 \%)$ & $94(68 \%)$ & 0.42 \\
\hline $200-299$ & $158(24 \%)$ & $87(23 \%)$ & $71(24 \%)$ & 0.66 & $32(23 \%)$ & $38(28 \%)$ & 0.40 \\
\hline$\geq 300$ & $29(4 \%)$ & $20(5 \%)$ & $9(3 \%)$ & 0.17 & $4(3 \%)$ & $6(4 \%)$ & 1 \\
\hline Mean GCS score & - & $7.0 \pm 3.0$ & $7.0 \pm 2.9$ & 0.78 & $7.2 \pm 2.9$ & $6.9 \pm 2.8$ & 0.41 \\
\hline $3-4$ & $215(31 \%)$ & $126(32 \%)$ & $89(30 \%)$ & 0.58 & $39(28 \%)$ & $37(26 \%)$ & 0.89 \\
\hline $5-8$ & $300(44 \%)$ & $165(42 \%)$ & $135(46 \%)$ & 0.34 & $67(48 \%)$ & $73(52 \%)$ & 0.54 \\
\hline $9-13$ & $172(25 \%)$ & $101(26 \%)$ & $71(24 \%)$ & 0.61 & $35(25 \%)$ & $31(22 \%)$ & 0.67 \\
\hline \multicolumn{8}{|l|}{ Pupil abnormality } \\
\hline Unilateral & $162(24 \%)$ & $80(21 \%)$ & $82(28 \%)$ & 0.03 & $27(19 \%)$ & $35(25 \%)$ & 0.32 \\
\hline Bilateral & $91(13 \%)$ & $64(16 \%)$ & $27(9 \%)$ & 0.006 & $16(11 \%)$ & $13(9 \%)$ & 0.65 \\
\hline$T \& D$ & $132(21 \%)$ & $76(21 \%)$ & $56(20 \%)$ & 0.76 & $25(20 \%)$ & $30(22 \%)$ & 0.76 \\
\hline tSAH & $484(71 \%)$ & $259(66 \%)$ & $225(78 \%)$ & 0.001 & $96(69 \%)$ & $99(73 \%)$ & 0.71 \\
\hline IVH & $84(12 \%)$ & $43(11 \%)$ & $41(14 \%)$ & 0.26 & $16(11 \%)$ & $23(16 \%)$ & 0.32 \\
\hline \multicolumn{8}{|l|}{ Basal cistern } \\
\hline Compressed & $261(38 \%)$ & $139(36 \%)$ & $122(42 \%)$ & 0.12 & $56(40 \%)$ & $52(37 \%)$ & 0.72 \\
\hline Absent & $164(24 \%)$ & $95(24 \%)$ & $69(24 \%)$ & 0.78 & $29(21 \%)$ & $27(19 \%)$ & 0.77 \\
\hline \multicolumn{8}{|l|}{ Midline shift (mm) } \\
\hline $0-4$ & $353(52 \%)$ & $199(52 \%)$ & $154(53 \%)$ & 0.73 & $76(55 \%)$ & $75(53 \%)$ & 0.90 \\
\hline $5-10$ & $142(21 \%)$ & $84(22 \%)$ & $58(20 \%)$ & 0.56 & $30(22 \%)$ & $29(21 \%)$ & 1 \\
\hline$>10$ & $182(27 \%)$ & $103(27 \%)$ & $79(27 \%)$ & 0.89 & $33(24 \%)$ & $36(26 \%)$ & 0.79 \\
\hline \multicolumn{8}{|l|}{ NTCDB CT classification } \\
\hline DI-I, DI-II & $115(17 \%)$ & $77(20 \%)$ & $38(13 \%)$ & 0.02 & $31(22 \%)$ & $25(18 \%)$ & 0.44 \\
\hline DI-III, DI-IV & $57(8 \%)$ & $25(6 \%)$ & $32(11 \%)$ & 0.04 & $11(8 \%)$ & $12(9 \%)$ & 1 \\
\hline
\end{tabular}


TABLE 1. Comparison of categorical and continuous variables for patients in the $\mathrm{C}$ group and TTM group before and after PS matching* (continued)

\begin{tabular}{|c|c|c|c|c|c|c|c|}
\hline \multirow[b]{2}{*}{ Parameter } & \multirow[b]{2}{*}{ Total } & \multicolumn{3}{|c|}{ Before PS Matching } & \multicolumn{3}{|c|}{ After PS Matching } \\
\hline & & C Group & TTM Group & p Value & C Group & TTM Group & $\mathrm{p}$ Value \\
\hline \multicolumn{8}{|c|}{ NTCDB CT classification (continued) } \\
\hline eML & $390(57 \%)$ & $206(53 \%)$ & $187(63 \%)$ & 0.004 & $81(57 \%)$ & $78(55 \%)$ & 0.80 \\
\hline Non-eML & $125(18 \%)$ & $84(21 \%)$ & $38(13 \%)$ & 0.004 & $18(13 \%)$ & $26(18 \%)$ & 0.26 \\
\hline \multicolumn{8}{|l|}{ Surgery } \\
\hline None & $226(33 \%)$ & $155(40 \%)$ & $71(24 \%)$ & $<0.001$ & $46(33 \%)$ & $46(33 \%)$ & 1 \\
\hline $\mathrm{BH}$ & $101(15 \%)$ & $64(16 \%)$ & $37(13 \%)$ & 0.17 & $18(13 \%)$ & $22(16 \%)$ & 0.62 \\
\hline Craniotomy & $341(50 \%)$ & $162(41 \%)$ & $179(61 \%)$ & $<0.001$ & $73(52 \%)$ & $71(50 \%)$ & 0.90 \\
\hline Other & $19(3 \%)$ & $11(3 \%)$ & $8(3 \%)$ & 0.94 & $4(3 \%)$ & $2(1 \%)$ & 0.69 \\
\hline \multicolumn{8}{|l|}{ ICU management } \\
\hline Sedative drugs & $337(49 \%)$ & $120(31 \%)$ & $217(74 \%)$ & $<0.001$ & $81(57 \%)$ & $74(52 \%)$ & 0.36 \\
\hline Analgesic drugs & $125(18 \%)$ & $33(8 \%)$ & $92(31 \%)$ & $<0.001$ & $24(17 \%)$ & $28(20 \%)$ & 0.63 \\
\hline NMBDs & $88(13 \%)$ & $20(5 \%)$ & $68(23 \%)$ & 0.04 & $15(11 \%)$ & $11(8 \%)$ & 0.50 \\
\hline AEDs & $269(39 \%)$ & $120(31 \%)$ & $149(51 \%)$ & $<0.001$ & $55(39 \%)$ & $68(48 \%)$ & 0.15 \\
\hline Diuretic drugs & $320(47 \%)$ & $136(35 \%)$ & $184(62 \%)$ & $<0.001$ & $68(48 \%)$ & $68(48 \%)$ & 1 \\
\hline ICP monitor placed & $229(33 \%)$ & $67(17 \%)$ & $162(55 \%)$ & $<0.001$ & $47(33 \%)$ & $55(39 \%)$ & 0.27 \\
\hline Mean AIS head & - & $4.3 \pm 1.0$ & $4.6 \pm 1.0$ & 0.002 & $4.4 \pm 0.9$ & $4.5 \pm 0.9$ & 0.23 \\
\hline Mean ISS & - & $25 \pm 12$ & $27 \pm 11$ & 0.15 & $26 \pm 12$ & $26 \pm 12$ & 0.79 \\
\hline
\end{tabular}

— = not applicable; $\mathrm{ABP}=$ arterial blood pressure; $\mathrm{BH}=$ bur hole; $\mathrm{RR}=$ respiratory rate.

* Mean values expressed as the mean \pm standard deviation.

no differences between clinical outcomes or mortalities in patients who were randomly assigned to undergo either TN (core temperature maintained under $37^{\circ} \mathrm{C}$ ) or $\mathrm{TH}$ (maintained at $\left.33^{\circ} \mathrm{C}\right) .^{6,7}$ However, there are no previous studies comparing clinical outcomes of patients who underwent TTM of either kind (TN or TH) and patients who did not undergo any type of TTM. This situation provided the impetus for the present study. Although, according to the
JNTDB, TH is defined as maintaining a core body temperature of $33^{\circ} \mathrm{C}-35^{\circ} \mathrm{C}$, and $\mathrm{TN}$ is defined as maintaining a body temperature of $35^{\circ} \mathrm{C}-37^{\circ} \mathrm{C}$, no specific target temperature is listed for either group. Thus, we suspect that the various medical facilities might have used different definitions for TH and TN. Accordingly, the TTM group in this study includes patients who underwent either $\mathrm{TN}$ or $\mathrm{TH}$, so that patients who underwent TTM at any temperature
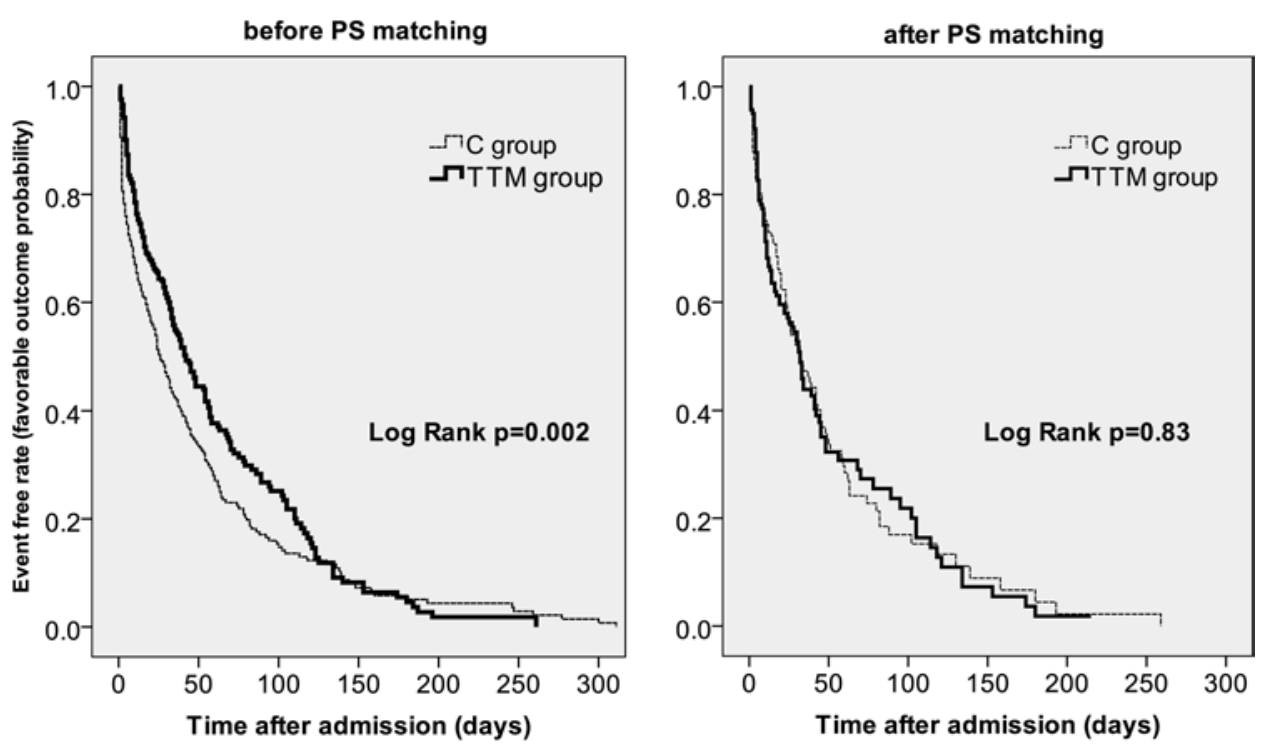

FIG. 2. Kaplan-Meier cumulative probability of favorable neurological outcome before (left) and after (right) PS matching. Bold solid line indicates patients who underwent TTM. Dotted line indicates the $\mathrm{C}$ group of patients who did not undergo TTM. 

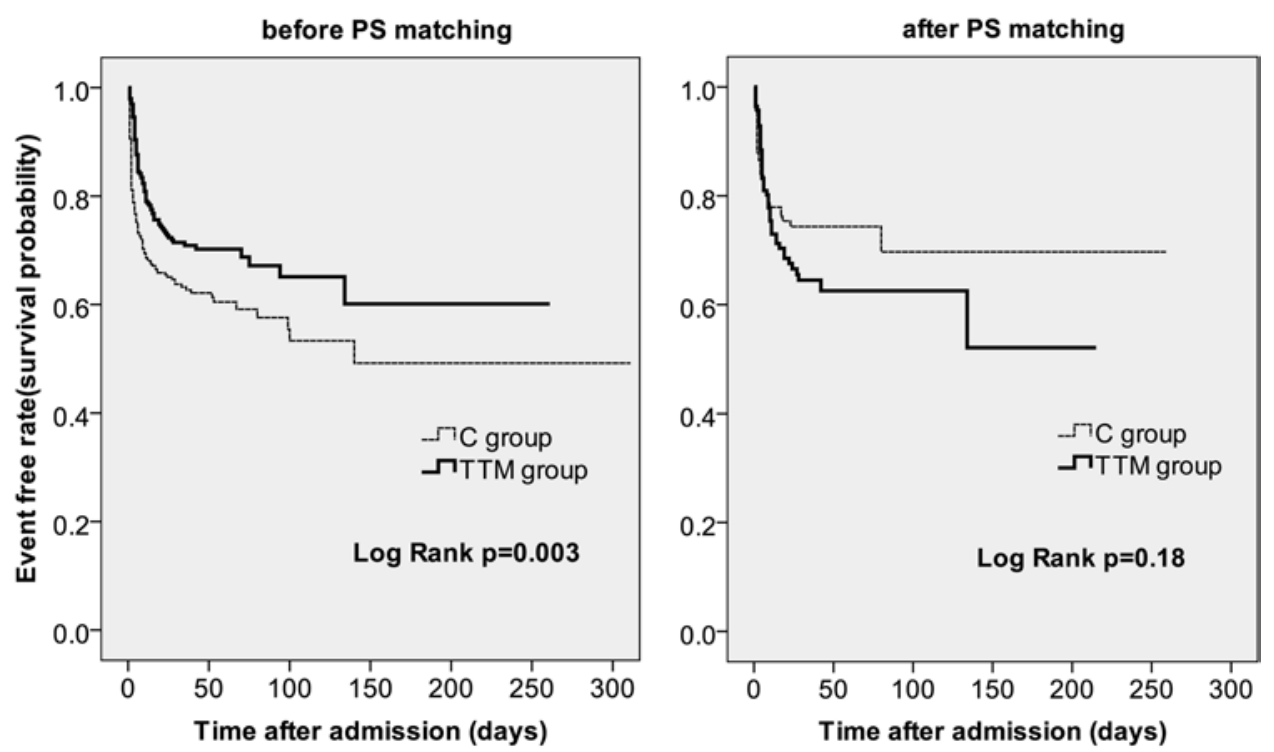

FIG. 3. Kaplan-Meier survival curve of patients in the TTM group as compared with patients in the $C$ group with regard to mortality before (left) and after (right) PS matching. Bold solid line indicates patients who underwent TTM. Dotted line indicates the $\mathrm{C}$ group.

were compared with patients who did not undergo TTM. Furthermore, patients with mild GCS scores (14-15) upon hospital arrival were excluded from the study, while those with moderate GCS scores (9-13) were included. Many patients with moderate TBI symptoms who had been able to talk during transport to the hospital or soon after arrival later underwent a marked deterioration and eventually entered into a comatose state. These so-called T\&D patients compose $25 \%$ of all cases. Given that so many severe cases may initially appear moderate, moderate cases cannot be ignored if we are to achieve a thorough understanding of TBI.

The only previous multivariate analysis that categorized data bank patients according to TTM status, a study by Suehiro et al., ${ }^{24}$ is limited to patients with surgically removed lesions (patients had GCS scores of 4-8; all patients with a GCS score of 3 or bilateral fixed and dilated pupils were excluded). In that study, TH was an independent factor related to favorable outcome. However, only 6 covariates were included: patient age (older or younger than 75 years), sex, GCS category (above or below a score of 6), pupillary findings, ISS, and ICP sensor placement. Other confounding factors that affect clinical outcomes were left out, including the potential selection bias for TTM and the problem of distinguishing patients designated as "do not attempt resuscitation (DNAR)." In our study, the patients in the TTM group were relatively young, quickly transported to advanced critical care and emergency centers, and presented with unilateral pupil dilation. They often underwent TTM as part of a multimodal treatment regimen that also included surgery. In contrast, the patients in the $\mathrm{C}$ group were more likely to be older and have bilateral pupil dilation and space-occupying lesions that could not be surgically removed. Few individuals in the $\mathrm{C}$ group received pharmacotherapy or surgical intervention; it is possible that the $\mathrm{C}$ group contained many very severe cases. Therefore, we could not ignore the possibility of selection bias for inclusion in the TTM group. We speculate that many patients in the TTM group received multimodal therapy intervention, whereas the $\mathrm{C}$ group included many DNAR patients who had declined such intervention. Given the raw data, however, it is difficult to make a clear distinction between patients who received intentional treatment intervention and the DNAR patients who did not. Therefore, our study attempted to match the characteristics of the 2 groups using a wide array of factors including patient characteristics, type of medical facility, severity of trauma, neurological intensive care, use of pharmacotherapy, surgical intervention, and so forth. The results of PS matching revealed no statistically significant differences in rates of poor outcome or mortality between the 2 groups.

PS analysis is a statistical method used to create a pseudorandom assignment for research data from observational studies in which random assignment is not possible. ${ }^{2}$ In simplified terms, it involves calculating a single variable (i.e., PS) from several covariates, searching for " 2 of a

TABLE 2. Poor neurological outcome and death at hospital discharge before and after PS matching in the TTM group*

\begin{tabular}{|c|c|c|c|c|}
\hline \multirow[b]{2}{*}{ Outcome } & \multicolumn{2}{|c|}{ Before PS Matching ( $n=687$ patients) } & \multicolumn{2}{|c|}{ After PS Matching ( $n=282$ patients) } \\
\hline & $\operatorname{HR}(95 \% \mathrm{Cl})$ & $\mathrm{p}$ Value & $\operatorname{HR}(95 \% \mathrm{Cl})$ & $\mathrm{p}$ Value \\
\hline Poor neurological outcome & $0.76(0.63-0.91)$ & 0.003 & $1.03(0.78-1.36)$ & 0.83 \\
\hline Death & $0.68(0.52-0.88)$ & 0.004 & $1.34(0.87-2.07)$ & 0.18 \\
\hline
\end{tabular}

\footnotetext{
* Cox proportional-hazards analysis.
} 
TABLE 3. Poor neurological outcome and death after PS matching for patients in the TTM group*

\begin{tabular}{|c|c|c|c|c|c|}
\hline \multirow[b]{2}{*}{ Category } & \multirow{2}{*}{$\begin{array}{c}\text { No. of } \\
\text { Patients }\end{array}$} & \multicolumn{2}{|c|}{ Poor Neurological Outcome } & \multicolumn{2}{|c|}{ Death } \\
\hline & & $\mathrm{HR}(95 \% \mathrm{Cl})$ & $p$ Value & $\mathrm{HR}(95 \% \mathrm{Cl})$ & p Value \\
\hline \multicolumn{6}{|l|}{ Age (yrs) } \\
\hline$<65$ & 138 & $1.22(0.76-1.94)$ & 0.41 & $1.19(0.54-2.60)$ & 0.67 \\
\hline$>65$ & 144 & $0.96(0.67-1.37)$ & 0.82 & $1.38(0.82-2.33)$ & 0.23 \\
\hline \multicolumn{6}{|l|}{ GCS score } \\
\hline $3-4$ & 76 & $0.93(0.56-1.52)$ & 0.76 & $1.35(0.67-2.71)$ & 0.40 \\
\hline $5-8$ & 140 & $1.25(0.82-1.90)$ & 0.30 & $1.78(0.88-3.63)$ & 0.11 \\
\hline $9-13$ & 66 & $0.72(0.39-1.32)$ & 0.29 & $0.72(0.27-1.90)$ & 0.51 \\
\hline \multicolumn{6}{|c|}{ NTCDB CT classification } \\
\hline $\mathrm{DI}$ & 79 & $1.09(0.62-1.91)$ & 0.76 & $1.23(0.53-2.85)$ & 0.63 \\
\hline eML & 159 & $0.83(0.57-1.21)$ & 0.33 & $1.31(0.72-2.38)$ & 0.38 \\
\hline Non-eML & 44 & $2.09(0.93-4.67)$ & 0.07 & $2.04(0.70-5.92)$ & 0.19 \\
\hline
\end{tabular}

kind" data, and then comparing these pairs in 2 groups. In this study we adopted a nearest-neighbor matching method that matches 1 estimated variable in the treatment group with the nearest variable in the control group. This method was adopted from previous reports, including observational studies on patients with cardiac arrest or TBI in the field of emergency medicine. ${ }^{4,14}$ There is no strong evidence that targeted temperature management improves the outcomes of patients with severe TBI, and it may be a second-tier therapy for intracranial hypertension resistant to surgery, or any intensive therapy. The 29 covariates that were chosen for calculating the PS in our study were deliberately selected to include important representative items of patient data, including information related to patient characteristics, the mechanism of injury, the transportation method, the institutional standards, findings on admission, CT findings, and acute-phase treatment (surgery, neurocritical care).

\section{Patient Age}

One explanation for the nature of our results may be the progressively aging society of Japan. A major reason why our study was unable to show any advantage associated with TTM could be the relatively advanced mean age of the patients, which must have had a substantial effect on the types of trauma most often seen and the subsequent patient conditions. ${ }^{16,26}$ Effective treatment for severe TBI in elderly patients, who make up half of all TBI cases, has not yet been established. Old age is considered an independent predictor of a poor outcome. ${ }^{8,17}$ The mean patient age in the National Acute Brain Injury Study 2 (NABIS2) was between 26 and 31 years, and the rate of favorable neurological outcome was $40 \%-44 \%$. $^{7}$ In our study, the mean patient age in both groups was 60 years, and the rate of a favorable outcome was only $30 \%$; therefore, it might not be appropriate simply to compare the results of the 2 studies. Furthermore, the mean age in our study increased from 56 years before PS matching to 60 years after PS matching. It is possible that PS matching skewed the age distribution toward the elderly, affecting the outcome of the study. As the mean age of the TTM group increased, young patients with high chances of survival were excluded. Furthermore, elderly DNAR patients in the C group who had a low survival rate were excluded from matching, which might explain why no significant difference in clinical outcomes between the 2 groups was observed.

\section{Level of Consciousness}

After PS matching, TTM for patients with moderate TBI (GCS scores of 9-13) tended to decrease the risk of a poor outcome (HR $0.72,95 \%$ CI $0.39-1.32, \mathrm{p}=0.29$ ), but TTM offered no significant risk reduction for those patients with extremely severe TBI (GCS scores of 3-4) or severe TBI (GCS scores of 5-8; Table 3). It is possible that the effect of TTM depends on the severity of the head injury at hospital admission. Furthermore, the category for consciousness on hospital arrival was treated as a covariate; in the Cox proportional-hazards analysis, the risk of a poor outcome for patients with extremely severe TBI was significantly higher (HR 1.77, 95\% CI 1.227-2.64, $\mathrm{p}=$ 0.03 ) than it was for patients with moderate TBI (HR 1.0). However, a comparison between moderate and severe TBI did not reveal any significant differences (HR 1.06, 95\% CI 0.74-1.53, $\mathrm{p}=0.75$; not shown in the table). Patients who required surgical intervention or had an accelerated shift from moderate to severe TBI after admission were expected to have a poor prognosis, similar to the prognosis in patients who presented with severe TBI at hospital admission. As the majority of preceding studies have focused on TTM for severe TBI, more research is needed to examine the usefulness of TTM in patients with moderate TBI who have a risk of deterioration.

\section{Pathology of Head Injury}

Some studies on the surgical removal of space-occupying lesions suggest an improved outcome after the very early induction of $\mathrm{TH}$ as compared with TN.,20 However, in our study, although the results showed that TTM combined with surgical intervention tends to decrease the risk 
of poor outcome (HR 0.83, 95\% CI 0.57-1.21, $\mathrm{p}=0.33$ ), this tendency was not statistically significant (Table 3). By contrast, a subgroup analysis by Clifton et al., ${ }^{6}$ who examined the effects of TH on the diffuse type of TBI, showed no significant improvement in the risk of poor outcome or mortality. Shiozaki et al. ${ }^{22}$ have also reported no improvements after TH in patients with diffuse brain swelling who presented with treatment-resistant and malignant intracranial hypertension. In our study, after PS matching, severe diffuse injuries composed $30 \%$ of the total diffuse type, but clinical outcomes after TTM showed either no change or a worsening trend (Table 3). This led us to a similar conclusion that TTM does not enable improvement in such patients.

\section{Mortality}

It should be noted that the results for mortality rate were reversed before and after PS matching. Before PS matching, TTM appeared to reduce the chance of in-hospital death by $32 \%$; after PS matching, TTM appeared to increase it by $34 \%$ (Table 2).

Figure 3 right shows that no significant differences were found between the Kaplan-Meier curves after matching $(\mathrm{p}=0.18)$. Differences were observed, however, between the survival curves for the 2 experimental groups; as the cumulative survival rate of the TTM group shows a clear decrease, the cumulative survival rate of the $C$ group increases. It is possible that PS matching eliminated patients with a favorable prognosis from the TTM group and eliminated DNAR patients with a poor prognosis from the $\mathrm{C}$ group, which affected the results of the study.

There is insufficient scientific evidence to demonstrate that $\mathrm{TH}$ improves the prognosis of patients with severe TBI or in ICP control patients, and its use in these patient groups is therefore not recommended. ${ }^{25}$ Any study that analyzes the usefulness of TTM in patients with TBI should consider adverse events such as infections, coagulation abnormalities, and changes in drug metabolism. Furthermore, complications of TH can include circulatory depression, delayed recovery from anesthesia, increased length of stay in the ICU, and ventilator-associated pneumonia after prolonged mechanical ventilation. ${ }^{11,21}$ According to an NABIS2 report, ${ }^{7} \mathrm{TH}$ increases the frequency of complications and tends to contribute to the deterioration of clinical outcomes in patients with severe TBI. These findings show that groups containing predominantly elderly patients are at risk for both serious complications and for outcome deterioration when treated with TTM. However, a thorough study with a large number of patients will be necessary to confirm these results.

\section{Limitations}

Our study has several potential limitations. First, because the age of the patient population was skewed toward the elderly, the efficacy of TTM in younger patients could not be examined. In addition, this study included not only patients with isolated head injuries, but also patients with multiple traumas who had an ISS of 30 or higher. Trauma deaths due to causes other than TBI were excluded, but we cannot rule out the possibility that trauma to other body areas affected clinical outcomes. Secondly, recent evidence reviews for various conditions have recommended using the term "targeted temperature management" instead of less specific phrases such as "therapeutic mild hypothermia."19 Targeted temperature management is a general term for any temperature management method (including $\mathrm{TH}$ and $\mathrm{TN}$ ) that intentionally attempts to maintain a certain body temperature. TTM details (specific target temperature, body part used for measuring the temperature, timing, method, and duration of TTM, and so forth) were not included in the database. The actual conditions of TTM were unclear, as the timing and methods of TTM depended greatly on the institution. For this reason, the validity of TTM classification may be called into question, and the effect of TTM timing on outcomes could not be examined in our study. Thirdly, patient outcome was measured at the time of hospital discharge, with no longitudinal follow-up. Outcome data for trauma patients 6 months after injury could not be used as assessment items because of the large amount of missing data, making any investigation of medium-term or long-term prognosis difficult. For this reason, we chose to assess patient outcome at hospital discharge for the primary outcome. Comparing patient outcomes over a long-term follow-up period might have led to different results. Fourthly, the nonrandom nature of the study and the limitations of the propensity-matching method must be mentioned. If other variables that may significantly affect outcomes are not recorded in this study, confounding factors cannot be eliminated, and selection bias could be inevitable. If cooling begins soon after hospital admission, some covariates associated with ICU management and surgical treatment might be considered intermediate variables, so-called posttreatment variables. In other words, these intermediate variables follow the induction of TTM. A causal relationship between induction of TTM and patient outcome might be underestimated when the PS adjustment is performed using the intermediate variable as a covariate.

In addition, we deliberately modified each item, from quantitative data to categorical variables, and these classifications may have affected the results. For example, if more than 29 items were selected as variables to adjust between the 2 groups, patient attributes could have been more strictly adjusted between the 2 groups. To verify our results, it will be necessary to perform a prospective randomized controlled trial to confirm the data. Fifthly, head CT findings based on NTCDB classification were used, although these findings, which are time dependent, can vary greatly during the acute phase of injury. Furthermore, the lesions were not uniform, and heterogeneous pathophysiological conditions may exist at the same time. Therefore, it is possible that the pathology classifications used in this study were not appropriate. Levels of consciousness varied greatly depending on the time of assessment during the acute phase. Subgroup analysis showed that TTM tended to decrease the risk of poor outcome when the GCS score at hospital admission was 9-13 and the NTCDB classification was eML. The small number of patients included in this subgroup analysis might be 1 of the reasons why this study did not show any statistically significant benefits of TTM. A large-scale study is needed to investigate which types of TTM might be effective. 


\section{Conclusions}

We assessed the current situations of TTM and compared its effectiveness under various conditions using data from the nationwide TBI data bank in Japan. The age of the patient population was skewed toward the elderly. Large differences in baseline characteristics and clinical variables existed between patients who underwent TTM and those who did not. To account for this, we developed a PS for TTM and controlled for potential confounding and selection bias. Our results did not prove that initiating a TTM protocol improved the clinical outcomes of patients with moderate or severe TBI in this data bank. Although none of these tendencies were obviously significant, TTM tended to decrease the risk of a poor outcome for patients with moderate TBI and for patients who underwent surgical removal of lesions. Further research is necessary to elucidate the effects of TTM using specific target temperatures and to assess the optimal role for TTM in brainoriented treatment.

\section{Acknowledgments}

The JNTDB project was initiated in 1996 by the JNTDB committee of the Japan Society of Neurotraumatology (JSNT). This epidemiological study was the third national study following Project 1998 (a 3-year study with 1002 subjects) and Project 2004 (a 2-year project with 1101 patients). The results were examined in biannual meetings. Data were gathered by ADJUST Co., Ltd., and analyzed by the secretariat office. We thank the chairman of the JNTDB committee, Michiyasu Suzuki, for his consent to the current study, and the 22 collaborating clinical centers (Sapporo Medical University Hospital, Aomori Prefectural Central Hospital, Sendai City Hospital, Tokyo Medical University Ibaraki Medical Center, Chiba Cardiovascular Center, Chiba Emergency Medical Center, The Jikei University Kashiwa Hospital, Tokyo Medical University Hospital, Nippon Medical School Hospital, Nihon University Itabashi Hospital, Teikyo University Hospital, Showa University Hospital, National Disaster Medical Center, Japanese Red Cross Musashino Hospital, St. Marianna University Hospital, Tokai University Hospital, Juntendo University Shizuoka Hospital, Saiseikai Shiga Hospital, Kyoto Kujo Hospital, Kagawa University Hospital, Yamaguchi University Hospital, Kurume University Hospital, Saiseikai Fukuoka Hospital, and Japanese Red Cross Kumamoto Hospital) for supplying the clinical data.

\section{References}

1. Arrich J, Holzer M, Havel C, Müllner M, Herkner H: Hypothermia for neuroprotection in adults after cardiopulmonary resuscitation. Cochrane Database Syst Rev 9:CD004128, 2012

2. Austin PC: An introduction to propensity score methods for reducing the effects of confounding in observational studies. Multivariate Behav Res 46:399-424, 2011

3. Bratton SL, Chestnut RM, Ghajar J, McConnell Hammond FF, Harris OA, Hartl R, et al: Guidelines for the management of severe traumatic brain injury. III. Prophylactic hypothermia. J Neurotrauma 24 (Suppl 1):S21-S25, 2007 (Erratum in Neurotrauma 25:276-278, 2008)

4. Chikuda H, Yasunaga H, Takeshita K, Horiguchi H, Kawaguchi H, Ohe K, et al: Mortality and morbidity after high-dose methylprednisolone treatment in patients with acute cervical spinal cord injury: a propensity-matched analysis using a nationwide administrative database. Emerg Med J 31:201-206, 2014

5. Clifton GL, Coffey CS, Fourwinds S, Zygun D, Valadka A,
Smith KR Jr, et al: Early induction of hypothermia for evacuated intracranial hematomas: a post hoc analysis of two clinical trials. J Neurosurg 117:714-720, 2012

6. Clifton GL, Miller ER, Choi SC, Levin HS, McCauley S, Smith KR Jr, et al: Lack of effect of induction of hypothermia after acute brain injury. N Engl J Med 344:556-563, 2001

7. Clifton GL, Valadka A, Zygun D, Coffey CS, Drever P, Fourwinds $S$, et al: Very early hypothermia induction in patients with severe brain injury (the National Acute Brain Injury Study: Hypothermia II): a randomised trial. Lancet Neurol 10:131-139, 2011

8. Flaada JT, Leibson CL, Mandrekar JN, Diehl N, Perkins PK, Brown AW, et al: Relative risk of mortality after traumatic brain injury: a population-based study of the role of age and injury severity. J Neurotrauma 24:435-445, 2007

9. Fox JL, Vu EN, Doyle-Waters M, Brubacher JR, Abu-Laban R, Hu Z: Prophylactic hypothermia for traumatic brain injury: a quantitative systematic review. CJEM 12:355-364, 2010

10. Gal R, Cundrle I, Zimova I, Smrcka M: Mild hypothermia therapy for patients with severe brain injury. Clin Neurol Neurosurg 104:318-321, 2002

11. Geurts M, Macleod MR, Kollmar R, Kremer PH, van der Worp HB: Therapeutic hypothermia and the risk of infection: a systematic review and meta-analysis. Crit Care Med 42:231-242, 2014

12. Jacobs SE, Berg M, Hunt R, Tarnow-Mordi WO, Inder TE, Davis PG: Cooling for newborns with hypoxic ischaemic encephalopathy. Cochrane Database Syst Rev 1:CD003311, 2013

13. Jiang JY, Gao GY, Li WP, Yu MK, Zhu C: Early indicators of prognosis in 846 cases of severe traumatic brain injury. $\mathbf{J}$ Neurotrauma 19:869-874, 2002

14. Karamanos E, Talving P, Skiada D, Osby M, Inaba K, Lam $\mathrm{L}$, et al: Is prehospital endotracheal intubation associated with improved outcomes in isolated severe head injury? A matched cohort analysis. Prehosp Disaster Med 29:32-36, 2014

15. Maekawa T, Yamashita S, Nagao S, Hayashi N, Ohashi Y: Prolonged mild therapeutic hypothermia versus fever control with tight hemodynamic monitoring and slow rewarming in patients with severe traumatic brain injury: a randomized controlled trial. J Neurotrauma 32:422-429, 2015

16. Masson F, Thicoipe M, Aye P, Mokni T, Senjean P, Schmitt $\mathrm{V}$, et al: Epidemiology of severe brain injuries: a prospective population-based study. J Trauma 51:481-489, 2001

17. Mosenthal AC, Lavery RF, Addis M, Kaul S, Ross S, Marburger R, et al: Isolated traumatic brain injury: age is an independent predictor of mortality and early outcome. $\mathbf{J}$ Trauma 52:907-911, 2002

18. Nielsen N, Wetterslev J, Cronberg T, Erlinge D, Gasche Y, Hassager C, et al: Targeted temperature management at $33^{\circ} \mathrm{C}$ versus $36^{\circ} \mathrm{C}$ after cardiac arrest. N Engl J Med 369:21972206, 2013

19. Nunnally ME, Jaeschke R, Bellingan GJ, Lacroix J, Mourvillier B, Rodriguez-Vega GM, et al: Targeted temperature management in critical care: a report and recommendations from five professional societies. Crit Care Med 39:1113-1125, 2011

20. Qiu W, Zhang Y, Sheng H, Zhang J, Wang W, Liu W, et al: Effects of therapeutic mild hypothermia on patients with severe traumatic brain injury after craniotomy. J Crit Care 22:229-235, 2007

21. Sandestig A, Romner B, Grände PO: Therapeutic hypothermia in children and adults with severe traumatic brain injury. Ther Hypothermia Temp Manag 4:10-20, 2014

22. Shiozaki T, Nakajima Y, Taneda M, Tasaki O, Inoue Y, Ikegawa $\mathrm{H}$, et al: Efficacy of moderate hypothermia in patients 
with severe head injury and intracranial hypertension refractory to mild hypothermia. J Neurosurg 99:47-51, 2003

23. Stocchetti N, Rossi S, Zanier ER, Colombo A, Beretta L, Citerio G: Pyrexia in head-injured patients admitted to intensive care. Intensive Care Med 28:1555-1562, 2002

24. Suehiro E, Koizumi H, Kunitsugu I, Fujisawa H, Suzuki M: Survey of brain temperature management in patients with traumatic brain injury in the Japan neurotrauma data bank. J Neurotrauma 31:315-320, 2014

25. Sydenham E, Roberts I, Alderson P: Hypothermia for traumatic head injury. Cochrane Database Syst Rev 15:CD001048, 2009

26. Tokutomi T, Miyagi T, Ogawa T, Ono J, Kawamata T, Sakamoto T, et al: Age-associated increases in poor outcomes after traumatic brain injury: a report from the Japan Neurotrauma Data Bank. J Neurotrauma 25:14071414, 2008

27. Yan Y, Tang W, Deng Z, Zhong D, Yang G: Cerebral oxygen metabolism and neuroelectrophysiology in a clinical study of severe brain injury and mild hypothermia. J Clin Neurosci 17:196-200, 2010

28. Zhi D, Zhang S, Lin X: Study on therapeutic mechanism and clinical effect of mild hypothermia in patients with severe head injury. Surg Neurol 59:381-385, 2003

\section{Disclosure}

This work was supported by a grant from the General Insurance Association of Japan.

\section{Author Contributions}

Conception and design: Miyata, Uemura. Acquisition of data: Miyata. Analysis and interpretation of data: Miyata. Drafting the article: Miyata, Ohnishi, Mikami, Wanibuchi, Uemura, Tanno. Critically revising the article: Maekawa, Mikami, Akiyama, Iihoshi, Uemura, Tanno. Reviewed submitted version of manuscript: Ohnishi. Statistical analysis: Miyata, Ohnishi, Maekawa. Study supervision: Mikuni, Narimatsu, Asai.

\section{Correspondence}

Kei Miyata, Department of Neurosurgery, Sapporo Medical University, South 1 West 16, Chuo-ku, Sapporo 060-8543, Japan. email: miyata@sapmed.ac.jp. 